\title{
Microbial Spectrum and Antibiotics Susceptibility Profile of Nosocomial Urinary Tract Infections in Mansoura Oncology Center
}

\author{
Saly S. E. Gomaa, Noha B. EL-Mashad, Douaa R. EL-Deeb and Sara G. Essa* \\ Department of clinical pathology, Mansoura Faculty of Medicine, Mansoura, Egypt \\ *Corresponding author
}

\section{A B S T R A C T}

\section{Keywords}

Hospital acquired urinary tract infection,

Chromogenic

Uriselect 4, Vitek system,

Antibiogram

Article Info

Accepted:

16 November 2020

Available Online:

10 December 2020
Hospital acquired infections (HAIs) are the commonest untoward incident in healthcare delivery worldwide that not only bring additional medical cost but also, extend hospital stay and affect patient's morbidity and mortality. Urinary tract infections (UTIs) account for majority of these infections. The objective is to estimate hospital acquired UTIs prevalence and antibiogram among the admitted cancer patients. Also, to evaluate the performance of chromogenic Uriselect 4 agar medium in isolation and presumptive identification of nosocomial UTIs. Among 10414 patients stayed in hospital > $48 \mathrm{~h}$., 1080patients were suspected to have nosocomial UTIs. $698(64.6 \%)$ were catheterized and $382(35.4 \%)$ were not. (4.49\%) patients confirmed to have nosocomial UTIs. $468(43.3 \%)$ yielded aerobic growth on CLED agar, versus $624(57.8 \%)$ on the chromogenic Uriselect4 media. On CLED, 408 isolates were identified by automated Vitek 2 system versus 348 $(33.3 \%)$ were identified by manual biochemical reactions while $10 \%$ and $1.7 \%$ of the isolates were not identified by manual biochemical tests and automated Vitek2 system respectively. E.coli was the most frequent isolated uropathogen in cancer patients identified by manual biochemical reactions, automated Vitek 2 system and chromogenic Uriselect 4 medium(11.1\%, 23.1\% and $17.8 \%$ respectively).Catheterization had been the main risk factor for nosocomial infection in our patients.

\section{Introduction}

Nowadays, Hospital acquired infections (HAIs) are the major worldwide safety concern for both patients and health-care workers (Scherbaum et al., 2014). HAIs monitored closely by agencies such as CDC to minimize their occurrence and improve patient safety (Monegro and Regunath, 2018). Hospital acquired infections occurs within 48 and 72 hours of patient's hospital admission (Rose et al., 2019). UTIs are estimated to be
25-40\% of HAIs so they represent the most common nosocomial infections (Pragash et al., 2017). Catheter associated urinary tract infection (CAUTI) represent one of the highest rates of occurrence among hospitalized patients, comprising about $36 \%$ of HAIs (Aly et al., 2016). Hence, urinary tract instrumentation and prolonged hospital stay are predisposing factors for developing nosocomial UTIs (Gharaghani et al., 2018). The predominant causative agents are Escherichia coli, Klebsiella spp., Proteus 
mirabilis, Enterococcus spp. and Staphylococcus (Xiaoyan et al., 2017). Identification of causative organism is crucial to plan for treatment protocol and decrease the risk of nosocomial infection. Our objective is to estimate the prevalence and antibiogram of nosocomial UTIs in the admitted cancer patients. Also, the performance of chromogenic Uriselect 4 agar medium which was compared with cysteine lactose electrolyte deficient medium (CLED) in isolation and presumptive identification of nosocomial UTIs.

\section{Materials and Methods}

This study was conducted on 1080 specimens that obtained from patients suspected to have nosocomial UTIs (64.6\% were catheterized, $35.4 \%$ were non-catheterized) from a total of 2304 urine samples received in OCMU laboratory from 10414 patients stayed more than $48 \mathrm{~h}$ from a total number of 49585 patients admitted to OCMU during the period from January 2019 to December 2019.

$\mathrm{Pd}$ (patient days): total patient's days number in hospital within this period $=24414$. Dd (Device days): total number of exposure to the devices for all patients during this period $=22757$

Symptomatic nosocomial UTIs were diagnosed based on the National Health Safety Network guidelines (NHSN, 2015). They include at least one of these signs or symptoms that affect patients without any recognized cause: fever more than $38^{\circ} \mathrm{C}$, urgency, frequency, or suprapubic tenderness and positive urine culture more than $10^{5}$ $\mathrm{cfu} / \mathrm{ml}$.

Early morning midstream voided urine or catheter-catch urine samples were aseptically collected in sterilized cap. Samples were then transported to microbiological laboratory within 2 hours.
Allurine specimens were cultured on both CLED agar and chromogenic Uriselect 4 media simultaneously then incubated at $37^{\circ} \mathrm{C}$. Significant growth on both plates was identified.

Presumptive bacterial growth identification was done on chromogenic Uriselect 4 medium according to their colour and colony morphology as shown by manufacture supplement catalogue (as shown in Photos A to K). Colonies on CLED agar were identified by gram stain, manual biochemical reactions such as; coagulase, catalase, citirate utilization, ureas, TSI and oxidase and confirmed by by automated Vitek 2 System. Tests for antimicrobial susceptibility were done on Mueller-Hinton agar according to Kirby-Bauer disk diffusion method. Nitrofurantoin, Cefepime, AmpicillinSulbactam, Ceftazidime, Cefoxitin, Ceftriaxone, Ciprofloxacin, Levofloxacin, Ofloxacin, Amoxicillin-Clavulanic, Norfloxacin and Cefotaxime were antimicrobial agents used.

\section{Results and Discussion}

Patients included in this study were 444males and 636 females, with range of age from 31 to 66 years.

Calculated equations were performed based on (Angshuman et al., 2015).

Among 1080 urine samples 468(43.3\%) patients confirmed to have nosocomial UTI, among them 306/468 (65.3\%) were CAUTI and 162/468 (34.7\%) were not CAUTI. Incidence density (CAUTI rate) was $13.44 / 1,000$ catheter days. DAI rate was $28.33 \%$, but HAIs rate was $4.49 \%$ (Table 1).

Most nosocomial UTIs are associated with urinary catheters (Babamahmoodi et al., 2015). Catheterized patients show greater risk for developing urinary tract infection (2.6 
times) more than non-catheterized patients (Melaku et al., 2012).

Another study reported that the incidence rate for device associated infections (DAI) was $19.55 \%$ (113/578). This is much lower than our findings. Nevertheless, the device utilization was very high in case of urinary catheter (0.838) in the same study which agrees with our results. DAI rate/100 cases was 6.23 while DAI/1000 cases was 4.25 (Jana et al., 2015).

Sawsan et al., 2020 reported the incidence rate of CAUTI was $29.5 \%$. The difference from the result could be due to the fact that this study included only cancer cases who were more liable to nosocomial UTI due to immunodeficiency. Moreover, patient days were much longer in the current study compared to others in the existing literature.

In this study, CLED agar culture revealed bacterial growth in 468 samples (43.3\%), gram negative bacilli were detected in 348 cases $(74.4 \%)$, whereas gram positive organisms were encountered in 120 cases (25.6\%).This findings were similar to the study conducted by Akter et al., 2014 which resulted in $(42.67 \%)$ bacterial growths and $(57.33 \%)$ showed no growth. Bitew et al., (2017) revealed that 256 of bacterial isolates were recovered, $68.4 \%$ of the isolates were Gram-negative and $31.6 \%$ isolates were Gram-positive bacteria. Furthermore, another study reported that gram negative bacteria accounted for $73.2 \%$ of cases with UTI, while the remaining cases were due to gram positive organisms (Yerega Belete et al., 2019).

(Table 2) represent the growth on chromogenic Uriselect 4 medium that yielded 792 isolates. The most frequent isolated organism among the gram-negative bacilli was E-coli 192(17.8\%), followed by Citrobacter 120 (11.1\%). Regarding gram positive organisms Enterococci 216 (20\%) were the most frequent.

In this study, (Table 3) shows the identification of organism growth was $43.3 \%$ and $57.8 \%$ in CLED agar and chromogenic Uriselect4 medium respectively. In addition, CLED agar yielded single organism in all specimens, while Uriselect4 detected mixed organisms in $19.2 \%$ of specimens. On comparing CLED agar with chromogenic Uriselect4 media, there was a significant difference between the two media in organism identification ( $p<0.001)$, this means that chromogenic Uriselect 4 media was superior to CLED agar in isolation and identification of UTI organisms.

There was good agreement between the two techniques. Uriselect 4 media had sensitivity and specificity of 100 and $74.5 \%$ respectively, with a diagnostic accuracy of $85.6 \%$. Perry et al., (2003) showed that chromogenic Uriselect4 medium lead to the recovery of $98.3 \%$ of strains, while it was $90.9 \%$ for CLED agar. This study agree with our findings that chromogenic Uriselect4 media were more effective in identification of uropathogens.

(Table 4), shows that $10 \%$ and $1.7 \%$ of the isolated organisms were not identified by manual biochemical tests and automated Vitek2 system respectively. The result indicated that E. coli was the most commonly isolated gram-negative bacteria $(11.1 \%)$ by manual biochemical test and $(23.1 \%)$ by vitek. This is due to the fact that, the commonest gastrointestinal tract and bowel flora is E. coli which ascends to urinary tract (Yerega Belete et al., 2019). 
Table.1 Device-associated infection rate, device utilization ratio, DAI/1000 patient days or 1000 device days

\begin{tabular}{|l|c|c|c|c|c|c|c|}
\hline Infection & $\begin{array}{c}\text { DAIs } \\
(\mathbf{n = 3 0 6 )}\end{array}$ & $\begin{array}{c}\text { Device } \\
\text { days(Dd) }\end{array}$ & $\begin{array}{c}\text { Patients } \\
\text { days(Pd) }\end{array}$ & $\begin{array}{c}\text { Device } \\
\text { utilization } \\
\text { ratio } \\
\text { (Dd/Pd) }\end{array}$ & $\begin{array}{c}\text { DAI } \\
\text { rate/100 } \\
\text { suspected } \\
\text { patients } \\
(\mathbf{n} / \mathbf{1 0 8 0})\end{array}$ & $\begin{array}{c}\text { DAI/1000 } \\
\text { device } \\
\text { days(incidence } \\
\text { density) }\end{array}$ & $\begin{array}{c}\text { HAIs } \\
\text { rate/100 } \\
\text { patients } \\
(\mathbf{n} / \mathbf{1 0 4 1 4}) \%\end{array}$ \\
\hline CAUTIs & 306 & 22757 & 24414 & 0.932 & 28.33 & 13.44 & 4.4 \\
\hline
\end{tabular}

Table.2 Phenotypic identification of the isolated organisms on chromogenic Uriselect 4 medium

\begin{tabular}{|c|c|c|c|c|}
\hline \multicolumn{3}{|c|}{ Isolated organisms on chromogenic Uriselect 4 media } & $\mathbf{N}$ & $\%$ \\
\hline \multirow[t]{6}{*}{ Gram negative Bacilli } & \multicolumn{2}{|l|}{ E-coli } & 192 & $17.8 \%$ \\
\hline & \multicolumn{2}{|l|}{ citrobacter } & 120 & $11.1 \%$ \\
\hline & \multicolumn{2}{|l|}{ Klebsiella } & 24 & $2.2 \%$ \\
\hline & \multicolumn{2}{|l|}{ Enterobacter } & 72 & $6.7 \%$ \\
\hline & \multirow{2}{*}{\multicolumn{2}{|c|}{$\begin{array}{c}\text { Serratia } \\
\text { Proteus }\end{array}$}} & 12 & $1.1 \%$ \\
\hline & Proteus & & 48 & $4.4 \%$ \\
\hline \multirow[t]{3}{*}{ Gram positive Cocci } & \multirow[t]{2}{*}{ Enterococei } & & 96 & $8.9 \%$ \\
\hline & & E-faccium & 96 & $\begin{array}{l}8.9 \% \\
2.2 \%\end{array}$ \\
\hline & \multicolumn{2}{|l|}{ Staphylococei } & 96 & $8.9 \%$ \\
\hline Yeast & \multicolumn{2}{|l|}{ Candida } & 12 & $1.1 \%$ \\
\hline
\end{tabular}

Table.3 Urine culture on CLED agar versus chromogenic Uriselect 4 medium

\begin{tabular}{|c|c|c|c|c|c|c|}
\hline & & \multicolumn{4}{|c|}{$\begin{array}{c}\text { Samples } \\
\mathbf{N}=\mathbf{1 0 8 0}\end{array}$} & \multirow{3}{*}{$\boldsymbol{P}$} \\
\hline & & \multicolumn{2}{|c|}{ CLED } & \multicolumn{2}{|c|}{ Uriselect 4} & \\
\hline & & $\mathbf{N}$ & $\%$ & $\mathbf{N}$ & $\%$ & \\
\hline \multirow[t]{4}{*}{ Culture } & No growth & 612 & $56.7 \%$ & 456 & $42.2 \%$ & \multirow{4}{*}{$<\boldsymbol{0 . 0 0 1}$} \\
\hline & Growth & 468 & $43.3 \%$ & 624 & $57.8 \%$ & \\
\hline & Single & 468 & $100 \%$ & 504 & $80.8 \%$ & \\
\hline & Mixed & O & $0 \%$ & 120 & $19.2 \%$ & \\
\hline
\end{tabular}

Table.4 Frequency of isolates by Manual biochemical identification and automated Vitek2 system

\begin{tabular}{|l|c|c|c|c|}
\hline & Manual biochemical tests & \multicolumn{2}{|c|}{ Vitek2 } \\
\hline E-coli & 120 & $11.1 \%$ & 108 & $23.1 \%$ \\
\hline Citrobacter & 84 & $7.8 \%$ & 96 & $20.5 \%$ \\
\hline Klebsiella & 24 & $2.2 \%$ & 24 & $5.1 \%$ \\
\hline Enterobacter & 12 & $1.1 \%$ & 36 & $7.7 \%$ \\
\hline Staph & 48 & $4.4 \%$ & 60 & $12.8 \%$ \\
\hline Enterococei & 60 & $5.6 \%$ & 72 & $15.4 \%$ \\
\hline Candida & 12 & $1.1 \%$ & 12 & $2.6 \%$ \\
\hline Unidentified & 108 & $10.0 \%$ & 8 & $1.7 \%$ \\
\hline
\end{tabular}


Table.5 Antimicrobial susceptibility pattern of the all isolated organisms (456) in this study

\begin{tabular}{|l|c|c|c|c|}
\hline \multirow{2}{*}{} & \multicolumn{4}{|c|}{$\mathbf{4 5 6}$ Isolates } \\
\cline { 2 - 5 } & \multicolumn{2}{|c|}{ Sensitive } & \multicolumn{2}{c|}{ Resistant } \\
\hline Levofloxacin & $\mathbf{N}$ & $\mathbf{\%}$ & $\mathbf{N}$ & $\mathbf{\%}$ \\
\hline Nitrofurantion & 368 & $80.7 \%$ & 88 & $19.3 \%$ \\
\hline Norfloxacin & 367 & $80.5 \%$ & 89 & $19.5 \%$ \\
\hline Ofloxacin & 346 & $75.9 \%$ & 110 & $24.1 \%$ \\
\hline Cefoxitin & 344 & $75.4 \%$ & 112 & $24.6 \%$ \\
\hline Ceftazidime & 332 & $72.8 \%$ & 124 & $27.2 \%$ \\
\hline Cefepim & 326 & $71.5 \%$ & 130 & $28.5 \%$ \\
\hline Ciprofloxacin & 307 & $67.3 \%$ & 149 & $32.7 \%$ \\
\hline Cefotaxime & 304 & $66.7 \%$ & 152 & $33.3 \%$ \\
\hline Ceftriaxone & 296 & $64.9 \%$ & 160 & $35.1 \%$ \\
\hline Amoxicillin.Clavulinic & 247 & $54.2 \%$ & 209 & $45.8 \%$ \\
\hline Ampicillin.Sulbactam & 146 & $32.02 \%$ & 310 & $67.98 \%$ \\
\hline
\end{tabular}

In the present study, the identified microorganisms by manual biochemical tests were E. coli 120 (11.1\%), followed by Citrobacter 84 (7.8\%), Klebsiella 24(2.2\%), and Enterobacter $12(1.1 \%)$. On the other hand, Enterococci were the commonest grampositive organism $60(5.6 \%)$, followed by Staphylococci 48 (4.4\%), and Candida 12 $(1.1 \%)$. In other study, culture-positive samples yielded a total of 199 bacterial isolates. E.coli was the leading bacteria isolated from samples $59.30 \%$ followed by Staph. Saprophyticus 19.09\%, Enterococcus spp.11.56\%, Klebseilla spp.5.53\%, Pseudomonas spp. 2.01\%, Proteus spp. 1.51\% and Enterobacter spp.1.00\% (Akter et al., 2014). In addition, other studies reported that E. coli $34.1 \%$ and Pseudomonas spp. $22.0 \%$ were the most isolated uropathogen. As regarding Gram-positive bacteria, S. saprophyticus $54.6 \%$ and S. aureus $27.3 \%$ were the commonest isolated bacteria (Yerega Belete et al., 2019).

By using automated Vitek2 system in this study, 408 isolates were identified E.coli, Citrobacter, and Klebsiella species were identified in $23.1 \%, 20.5 \%$, and $5.1 \%$ respectively. Enterococci, Staphylococcus, Enterobacter, and Candida were detected in $15.4 \%, 12.8 \%, 7.7 \%$ and $2.6 \%$ respectively, while $1.7 \%$ weren't identified by Vitek2 system.

Bitew et al., (2017) showed that Staphylococci were the commonest grampositive organism identified by automated Vitek2 system $21.4 \%$, followed by Enterococci $5.9 \%$, Streptococci $3.5 \%$ and kocuria $0.8 \%$. Regarding gram-negative species, E. coli $52.7 \%$ and K.pneumoniae $7 \%$. (Table 5), shows assessment of antimicrobial susceptibility pattern of the isolated organisms on Muller-Hinton agar by disc diffusion method. With exception of 12 Candida species isolates, the remaining 456 isolates revealed $80.7 \%$ sensitivity to Levofloxacin, $80.5 \%$ to Nitrofurantoin, $75.9 \%$ to Norfloxacin followed by $75.4 \%$ to Ofloxacin while Amoxicillin-Clavulinic and Ampicillin-Sulbactam were the most resistant.
Abdollahi et al., (2016) introduced carbapenem and third generation 
cephalosporins as the most effective antibiotics against pathogens recovered from Iranian cancer patients.

On evaluating the microbial susceptibility pattern of each organism showed a high rate of resistance $(>80 \%)$ to AmoxicillinClavulinic and Ampicillin-Sulbactam for most isolates. While E.coli was more sensitive to Cefoxitin $90 \%$, Nitrofurantoin and Levofloxacin $80 \%$, Citrobacter showed sensitivity to Ceftazidime 85.7 and Staphylococci showed sensitivity to Nitrofurantoin $93 \%$.

Other studies reported that Gram-negative bacterial sensitivity was high for ciprofloxacin, Norfloxacin and Ceftriaxone $70 \%, 63.4 \%, 60 \%$ respectively. And sensitivity for Gram-positive isolates were high for Ciprofloxacin 77.8\%, Penicillin 72.8\% and Erythromycin $72.8 \%$.Ampicillin, Penicillin and Cotrimoxazole were resistant with all isolated S. aureus. (Yerega Belete et al., 2019).

In conclusions: the rate of catheter associated urinary tract infections (CAUTI) as an important device associated healthcare acquired infections was $65.3 \%$.The most frequent gram negative uropathogen isolated in cancer patients was E.coli which was identified by manual biochemical reactions, automated Vitek 2 system and chromogenic Uriselect 4 media: $11.1 \%, 23.1 \%$ and $17.8 \%$ respectively.

On using automated Vitek 2 system, 8 (1.7\%) isolates weren't identified. Using disk diffusion method; Levofloxacin and Nitrofurantoin were the most sensitive antibiotics for CAUTI $80.7 \%$ and $80.5 \%$ respectively, while Ampicillin/Sulbactam and Amoxicillin/Clavulanic were the most resistant antibiotics $81.8 \%$ and $67.98 \%$ respectively. Chromogenic Uriselect 4 media is cost effective and superior to CLED agar in growth of 156 urine samples (14.4\%) and isolation of 120 mixed growths.

\section{References}

Abdollahi, A., Hakimi, F., Doomanlou, M. and Azadegan, A. (2016). Microbial and antibiotic susceptibility profile among clinical samples of patients with acute leukemia. International journal of hematology-oncology and stem cell research, 10(2), 61.

Akter L, Haque R and Salam MA. 2014. "Comparative evaluation of chromogenic agar medium and conventional culture system for isolation and presumptive identification of uropathogens".Pak J Med Sci. 30(5): 1033-8.

Aly SA, Tawfeek RA and Mohamed IS 2016."Bacterial catheter-associated urinary tract infection in the Intensive Care Unit of Assiut University Hospital". Al Azhar Assiut Medical Journal. 14:5258

Angshuman Jana, N.K. Pal, Arijit Majumdar, Jayeeta Mitra, Anirban Jana, Soumali Biswas, Babita Bag: Device-associated infection rates and median lengthof acquiring device-assotiated infection in an intensive therapeutic unit of an Indian hospital. Jornal of Medicine in the Tropics (2015) 17:2;97-102.

Babamahmoodi F, Ahangarkani F, Davoudi AR. 2015. "Hospital-Acquired Infections, Bacterial Causative Agents and Antibiotic Resistance Pattern In Intensive Care Units At Teaching Hospitals In North Of Iran". Int J Med Invest 4(1); 152-160.

Bitew, A., Molalign, T. and Chanie, M. 2017. "Species distribution and antibiotic susceptibility profile of bacterial uropathogens among patients complaining urinary tract infections". BMC infectious diseases, 17(1), 654.

Gharaghani M, Taghipour S, Halvaeezadeh M and Mahmoudabadi AZ. 2018. "Candiduria; a review article with specific 
data from Iran". Turkish Journal of Urology. 44(6):445-452.

Jana, A., Pal, N., Majumdar, A., Mitra, J., Jana, A., et al., 2015. "Device-associated infection rates and median length of acquiring device-associated infection in an intensive therapeutic unit of an Indian hospital".Journal of Medicine in the Tropics, 17(2), 97.

Melaku, S., Kibret, M., Abera, B. and GebreSellassie, S. 2012. "Antibiogram of nosocomial urinary tract infections in Felege Hiwot referral hospital, Ethiopia". Afr Health Sci, 12(2), 134-139. doi:10.4314/ahs.v12i2.9.

Monegro AF and Regunath H 2018. "Hospital Acquired Infections". StatPearls Publishing, Treasure Island (FL).(PMID:28722887)

National health safety network (NHSN). Patient safety components manual, Atlanta, Georgia: CDC (on line) available from: https://www.cdc.gov/nhsn/pdfs/validation /2015/pcs manual 2015 (Accessed on January, 2015)

Perry, J., Butterworth, L., Nicholson, A., Appleby, M. and Orr, K. 2003. "Evaluation of a new chromogenic medium, Uriselect 4, for the isolation and identification of urinary tract pathogens". Journal of clinical pathology, 56(7), 528531.

Pragash DS, Girija S, Sekar U, Rayapu V and Sheriff DS 2017. "Uropathogens and Diabetes Mellitus- a perspective". IOSR Journal of Dental and Medical Sciences (IOSR-JDMS). 16(5): 29-32.

Rosenthal, V., Todi, S., Alvarez-Moreno, C.,
Pawar, M., Karlekar, A., et al., 2012. "Impact of a multidimensional infection control strategy on catheter-associated urinary tract infection rates in the adult intensive care units of 15 developing countries: findings of the International Nosocomial Infection Control Consortium (INICC)". Infection, 40(5), 517-526.

Sawsan A. Omer, Fawkia E. Zahran, Ahmed Ibrahim, Lalia A. Sidahmed, Gamil Karam, Fasial Almulhim, Sabri A. Soltan 2020."Risk Factors for Catheter Associated Urinary Tract Infections (CAUTI) in Medical Wards and Intensive Care Units (ICU)". Journal of Microbiology Research, 10(1):1-5

Scherbaum M, Kösters K, Mürbeth RE, Ngoa UA, Kremsner PG, Lell B and Alabi A 2014. "Incidence, pathogens and resistance patterns of nosocomial infections at a rural hospital in Gabon". BMC Infectious Diseases 14:124.

Xiaoyan Li, Yunqin Chen, Weiguo Gao, Hao Ye, Zhongchao Shen, Zehuai Wen, and Jia Wei2017. "A 6-year study of complicated urinary tract infections in southern China: prevalence, antibiotic resistance, clinical and economic outcomes". Ther Clin Risk Manag. 13: 1479-1487.

Yerega Belete, D. A., Woldeamanuel, Y., Yihenew, G. and Gize, A. 2019. "Bacterial Profile And Antibiotic Susceptibility Pattern Of Urinary Tract Infection Among Children Attending Felege Hiwot Referral Hospital, Bahir Dar, Northwest Ethiopia". Infection and Drug Resistance, 12, 3575.

\section{How to cite this article:}

Saly S. E. Gomaa, Noha B. EL-Mashad, Douaa R. EL-Deeb and Sara G. Essa. 2020. Microbial Spectrum and Antibiotics Susceptibility Profile of Nosocomial Urinary Tract Infections in Mansoura Oncology Center. Int.J.Curr.Microbiol.App.Sci. 9(12): 2198-2204. doi: https://doi.org/10.20546/ijcmas.2020.912.259 